\title{
Classification-Important Step to Improve Management of Patients with an Open Abdomen
}

\author{
Martin Björck · Andreas Bruhin · Michael Cheatham • \\ Daniel Hinck · Mark Kaplan · Guiseppe Manca · \\ Thomas Wild $\cdot$ Alastair Windsor
}

Published online: 17 April 2009

(c) The Author(s) 2009. This article is published with open access at Springerlink.com

\begin{abstract}
This short report is a distillation of the proceedings from a consensus group meeting in January 2009. It outlines a proposed classification system for patients with an open abdomen (OA). The classification allows (1) a description of the patient's clinical course; (2) standardized clinical guidelines for improving OA management; and (3) improved reporting of OA status, which will facilitate comparisons between studies and heterogeneous patient
\end{abstract}

M. Björck ( $\bowtie)$

Department of Vascular Surgery, Institution of Surgical

Sciences, Uppsala University Hospital, Uppsala SE-751 85,

Sweden

e-mail: martin@bjorck.pp.se

\section{A. Bruhin \\ Department of Trauma and visceral surgery, Luzern, Switzerland}

\section{Cheatham}

Surgical Intensive Care Units, Orlando Medical Centre, Orlando, USA

D. Hinck

Klinik fur Visceralchirurgie, Itzehoe, Germany

\section{Kaplan}

Division of Trauma, Surgical Critical Care, Albert Einstein

Medical Center, Philadelphia, USA

G. Manca

Department of General and Vascular Surgery, Careggi Hospital, Florence, Italy

T. Wild

Universitätskikinik für Chirurgie, Medizinische Universität Wien, Vienna, Austria

A. Windsor

Department of General Surgery, University College Hospital, London, UK populations. The following grading is suggested: grade $1 \mathrm{~A}$, clean OA without adherence between bowel and abdominal wall or fixity of the abdominal wall (lateralization); grade $1 \mathrm{~B}$, contaminated $\mathrm{OA}$ without adherence/fixity; grade $2 \mathrm{~A}$, clean OA developing adherence/fixity; grade $2 \mathrm{~B}$, contaminated OA developing adherence/fixity; grade 3, OA complicated by fistula formation; grade 4 , frozen OA with adherent/fixed bowel, unable to close surgically, with or without fistula. We propose that this classification system will facilitate communication, clarify OA management, and potentially improve patient care.

\section{Introduction}

The knowledge that a tense abdomen is a life-threatening condition is very old. The pediatric surgeon Gross recognized the clinical importance of a "tense" abdomen in 1948 as a complication of the repair of large omphaloceles [1]. However, it was not until 1984 that the term "abdominal compartment syndrome" (ACS) was suggested by Kron et al. [2]. Recent publications of consensus documents from the World Society of the Abdominal Compartment Syndrome (WSACS) in 2006 and 2007 have refined the definitions [3] and guidelines for management of ACS [4].

Despite increased understanding of ACS, clinicians often face the problem of managing a patient with an open abdomen (OA). The patient may reach this situation through a number of clinical pathways: (1) the septic contaminated abdomen that cannot be closed because of infection and/or a second-look laparotomy is mandatory; (2) a tense abdomen after massive resuscitation or a prolonged major surgical procedure, at risk of developing 
ACS; (3) a "damage control" situation where the patient remains inadequately resuscitated and needs a period of intensive care therapy prior to a definitive surgical procedure; and (4) primary or secondary ACS, requiring lifesaving decompressive laparotomy. Much has been made in the literature of the underlying etiology of ACS and how it should guide therapy. More specifically, there seems to be some confusion regarding the treatment of patients with trauma versus sepsis as the primary clinical cause and whether management should therefore differ. It is important to realize that the fundamental underlying pathophysiologic process is the same for both scenarios, the only difference being the time line of the evolving pathologic process.

Managing the patient with an OA is a great challenge, even for an experienced clinician. Ivatury's review in this month's issue of the World Journal of Surgery [5] outlines the key therapeutic approaches to such patients. Some kind of temporary abdominal closure (TAC) is needed to protect the intestines, maintain a sterile or at least clean environment, and avoid fluid and temperature loss. Establishing what constitutes the best "dressing" in this situation remains a difficult and controversial challenge [6-11]. It seems evident, therefore, that there are a number of areas where the best patient management remains unclear. To help clarify some of these issues, the authors thought that a robust, simple clinical classification for describing the OA within this diverse and complex patient population would be useful.

We have focused on the $\mathrm{OA}$; but when discussing management, it must be remembered that the focus is the patient, not the abdomen. Nutrition, ambulation, and control of infection and the inflammatory reaction are important for the prognosis. Early enteral nutrition is probably beneficial [5]. These issues should also be addressed when evaluating various TACs and their respective costeffectiveness.

\section{Aims of the classification}

Discussions regarding classification were based on the work of Banwell and Téot [12] and Swan and Banwell [13] as well as a consensus meeting held in October 2007. The proposed classification system outlined here was designed to fulfill a number of purposes. It needed to describe the natural history of clinical deterioration among these patients from relatively simple to much more complex scenarios. In so doing, it would allow clinical guidelines to be established for the management of these various scenarios. The principal goals of these clinical guidelines would be to: (1) prevent further deterioration and escalation within the classification system resulting in a more complex OA; and (2) appropriately manage and maintain the patient's OA at the lowest and simplest grade to facilitate the ultimate goal of achieving primary delayed fascial closure as quickly as clinically appropriate. Such a classification system would additionally help standardize clinical reporting and thus allow units managing these patients to compare treatments and outcomes more easily and to be involved in studies across centers.

\section{Proposed classification system}

The proposed classification system is outlined in Table 1. The grades are briefly described here, with their proposed management discussed.

- Grade 1A: clean OA without adherence between bowel and abdominal wall or fixity (lateralization of the abdominal wall). This relatively simple scenario is common following a decompressive laparotomy for ACS after a ruptured abdominal aortic aneurysm or abdominal trauma not affecting the gastrointestinal (GI) tract. The patient may have other risk factors for poor outcome, but the prognosis regarding the $\mathrm{OA}$ is favorable. The aims of treatment are straightforward: to maintain a clean OA without adherence between the intestines and the abdominal wall, without lateralization of the abdominal wall, contamination or fistula formation, and, ultimately, to achieve primary delayed fascial closure.

- Grade 1B: contaminated OA without adherence/fixity. Typical scenarios are patients with perforated diverticulitis, anastomotic breakdown after colorectal surgery, or trauma affecting the GI tract. The aims of treatment are twofold: first, to move toward a lower grade within the classification system by transforming the OA into a clean situation (e.g., by deviating the fecal flow with a stoma); and second, to focus on preventing deterioration into a less favorable state by preventing adhesions, fixity, and fistulation.

Table 1 Proposed classification of the open abdomen

\begin{tabular}{ll}
\hline Grade & Description \\
\hline 1A & $\begin{array}{c}\text { Clean OA without adherence between bowel and abdominal } \\
\text { wall or fixity (lateralization of the abdominal wall) }\end{array}$ \\
1B & $\begin{array}{c}\text { Contaminated OA without adherence/fixity } \\
\text { 2A }\end{array}$ \\
Clean OA developing adherence/fixity
\end{tabular}

OA Open abdomen 
- Grade 2A: clean OA developing adherencelfixity. This patient may have been treated in a suboptimal fashion, having been grade $1 \mathrm{~A}$ or $1 \mathrm{~B}$ prior to becoming grade 2A. Adhesions have developed between the intestines and the abdominal wall, and/or the fascia is beginning to become fixed laterally. Primary delayed fascial closure now becomes difficult as a result of the initial management. Every effort should be made to prevent and/or reverse this situation, gently breaking down the adhesions and utilizing additional techniques to overcome the ensuing lateralization of the abdominal wall. Ideally, the patient should be converted to grade 1, if possible; but, equally, the aim of treatment is to prevent further deterioration to a less favorable situation by trying to minimize the abdominal wall defect and prevent fistulization. One option is to perform a partial fascial closure, perhaps combined with skin and subcutaneous tissue-only coverage or a combined mesh and split-thickness skin graft. Such procedures can result in good functional abdominal closure without substantial morbidity.

- Grade 2B: contaminated OA developing adherencel fixity. This patient may have a septic abdomen, where source control has not yet been achieved and where adhesions and/or fixity may preclude subsequent fascial closure. The aims of treatment are twofold: (1) control contamination so the patient improves to grade $2 \mathrm{~A}$ for later closure of the $\mathrm{OA}$ according to the principles outlined above; and (2) prevent further deterioration with development of an enteric fistula and/or of a completely frozen abdomen (grades 3-4).

- Grade 3: OA complicated by fistula formation. The development of an enteric fistula represents significant clinical deterioration in the patient with an OA. A number of techniques have been reported that may allow early closure or fistula control in this situation. It may be possible, and indeed preferable, to convert the patient to a lower OA grade and therefore to a simpler clinical scenario. Once again, the primary focus of treatment in this group is to minimize fascial lateralization and thus the subsequent fascial defect. In addition, attempts must be made to prevent the development of further adhesions and deterioration to grade $4 \mathrm{OA}$. Protecting the fascia and skin from deterioration is important in all grades of $\mathrm{OA}$, but with a fistula this concern is even greater. A TAC that permits deviation of intestinal contents is crucial for success.

- Grade 4: frozen OA with adherent/fixed bowel, unable to close surgically, with or without fistula. The key here is prevention. Early, appropriate intervention in the scenarios outlined above should prevent the fixed, frozen abdomen. The management of this situation (more often with fistulation) is well documented elsewhere and relies principally on returning the patient's physiology and nutrition to normal, protecting skin and fascia, and preventing sepsis. Ultimately, a complex reconstruction is required, usually at about 6 to 12 months.

\section{Conclusion}

We hope the OA classification system outlined above will contribute to the understanding, treatment, and ultimately the outcome of these patients. The authors recognize the complexity of the OA, and clearly there is much more that needs to be clarified with regard to etiology, pathophysiology, and management. The suggested classification system needs to be evaluated in prospective studies.

Acknowledgments The Consensus group meetings were sponsored by KCI, a company that provides the Abdominal VAC system. KCI had no influence on the content of this article. The authors have the following conflicts of interest relating to the KCI: M.B. received an unrestricted (unconditional) research grant in his role as a principal investigator of an ongoing multicenter study on the OA. M.B., M.C., D.H., M.K., and A.W. served as speakers at educational activities organized by the KCI.

Open Access This article is distributed under the terms of the Creative Commons Attribution Noncommercial License which permits any noncommercial use, distribution, and reproduction in any medium, provided the original author(s) and source are credited.

\section{References}

1. Gross R (1948) A new method for surgical treatment of omphaloceles. Surgery 24:277-292

2. Kron IL, Harmon PK, Nolan SP (1984) The measurement of intra-abdominal pressure as a criterion for abdominal re-exploration. Ann Surg 199:28-30

3. Malbrain MLNG, Cheatham ML, Kirkpatrick A et al (2006) Results from the International Conference Experts on IntraAbdominal Hypertension and Abdominal Compartment Syndrome. I. Definitions. Intensive Care Med 32:1722-1732

4. Cheatham ML, Malbrain MLNG, Kirkpatrick A et al (2007) Results from the International Conference Experts on IntraAbdominal Hypertension and Abdominal Compartment Syndrome. II Recommendations. Intensive Care Med 33:951-962

5. Ivatury RR (2009) Update on open abdomen management: achievements and challenges. World J Surg. doi:10.1007/ s00268-009-0005-7

6. Wittmann DH, Aprahamian C, Bergstein JM (1990) Etappenlavage: advanced diffuse peritonitis managed by planned multiple laparotomies utilizing zippers, slide fastener, and Velcro analogue for temporary abdominal closure. World J Surg 14:218-226

7. Brock WB, Barker DE, Burns RP (1995) Temporary closure of open abdominal wounds: the vacuum pack. Am Surg 61:30-35

8. Barker DE, Kaufmann HJ, Smith LA et al (2000) Vacuum pack technique of temporary abdominal closure: a 7-year experience with 112 patients. J Trauma 48:201-206 
9. Suliburk JW, Ware DN, Balogh Z et al (2003) Vacuum-assisted wound closure achieves early fascial closure in open abdomens after severe trauma. J Trauma 55:1155-1161

10. Miller PR, Meredith JW, Johnson JC et al (2004) Prospective evaluation of vacuum-assisted fascial closure after open abdomen: planned ventral hernia rate is substantially reduced. Ann Surg 239:608-616

11. Petersson U, Acosta S, Björck M (2007) Vacuum-assisted wound closure and mesh-mediated continuous traction: a novel combination of two techniques for closure of the open abdomen. World J Surg 31:2133-2137

12. Banwell PE, Téot L (2003) Topical negative pressure (TNP): the evolution of a novel wound therapy. J Wound Care 12:22-28

13. Swan MC, Banwell PE (2005) The open abdomen: aetiology, classification and current management strategies. J Wound Care 14:7-11 